Klaus Gantert

Erfolgreich recherchieren - Linguistik

De Gruyter Studium 


\section{Erfolgreich recherchieren}

Herausgegeben von

Klaus Gantert 
Klaus Gantert

Erfolgreich

recherchieren -

Linguistik

DE GRUYTER

SAUR 
ISBN 978-3-11-029900-7

e-ISBN 978-3-11-029901-4

ISSN 2194-3443

Library of Congress Cataloging-in-Publication Data

A CIP catalog record for this book has been applied for at the Library of Congress.

\section{Bibliografische Information der Deutschen Nationalbibliothek}

Die Deutsche Nationalbibliothek verzeichnet diese Publikation in der

Deutschen Nationalbibliografie; detaillierte bibliografische Daten

sind im Internet über http://dnb.dnb.de abrufbar.

(C) 2013 Walter de Gruyter GmbH, Berlin/Boston

Satz: Medien Profis GmbH, Leipzig

Druck und Bindung: Hubert \& Co. GmbH \& Co. KG, Göttingen

@ Gedruckt auf säurefreiem Papier

Printed in Germany

www.degruyter.com 


\title{
Vorwort
}

\author{
suchen wissen \\ ich was suchen \\ ich nicht wissen was suchen \\ ich nicht wissen wie wissen was suchen \\ ich suchen wie wissen was suchen \\ ich wissen was suchen \\ ich suchen wie wissen was suchen \\ ich wissen ich suchen wie wissen was suchen \\ ich was wissen \\ Ernst Jandl, die bearbeitung der mütze, 1978
}

Das Angebot an wissenschaftlicher Literatur und Fachinformation hat sich in den letzten Jahren in der Linguistik enorm vergrößert. Parallel zu dieser Entwicklung sind auch die Rechercheinstrumente, mit denen nach Fachliteratur und wissenschaftlichen Informationen gesucht werden kann, immer zahlreicher und leistungsfähiger geworden. In diesem Zuwachs liegt jedoch nicht nur ein Segen, sondern auch ein Fluch: Immer vielfältiger werden die Typen der Informationsressourcen, immer größer die Zahl der einschlägigen Datenbanken, immer unübersichtlicher die Treffermengen. Entsprechend wird es immer schwieriger, verlässlich alle Publikationen und Informationen $\mathrm{zu}$ einem Themengebiet $\mathrm{zu}$ finden, zu bewerten und sinnvoll für die eigene Arbeit zu verwenden.

Dieses Buch soll Ihnen helfen, den Überblick über die wichtigsten Informationsangebote für Sprachwissenschaftler zu bekommen und diese erfolgreich zu nutzen. Hierfür werden die grundlegenden Typen von Informationsressourcen mit ihren jeweiligen Stärken und Schwächen vorgestellt und die zentralen Angebote detailliert besprochen.

Nahezu alle Aspekte und Teilgebiete der Linguistik werden in diesem Band berücksichtigt - dies entspricht auch der inhaltlichen Ausrichtung der meisten linguistischen Informationsressourcen. Schwerpunkte des Buches liegen auf der Allgemeinen und Vergleichenden Sprachwissenschaft sowie auf der germanistischen, anglistischen und romanistischen Linguistik. Das Buch richtet sich in erster Linie an die Studierenden dieser Fächer. Es kann für konkrete eigene Rechercheaufgaben verwendet werden (z. B. für eine Seminar- oder Abschlussarbeit), zur selbstständigen Einarbeitung in das Gebiet der linguistischen Recherche oder auch zur Begleitung und Vertiefung entsprechender Schulungsveranstaltungen, die heute an nahezu allen wissenschaftlichen Bibliotheken angeboten werden.

Mit dem Bibliothekskatalog, den linguistischen Fachbibliographien und den Internetsuchmaschinen werden im ersten Teil des Buches (Basics) zunächst die wichtigsten und am häufigsten verwendeten Rechercheinstrumente ausführlich dargestellt. Der zweite Teil (Advanced) präsentiert mit weiteren Bibliographien, Zeitschriftenverzeichnissen, Fachportalen, Nachschlagewerken und Informationsressourcen zu den einzelnen Sprachen noch mehr Instrumente für eine vertiefte sprachwissenschaftliche Literatur- und Informationsrecherche. 


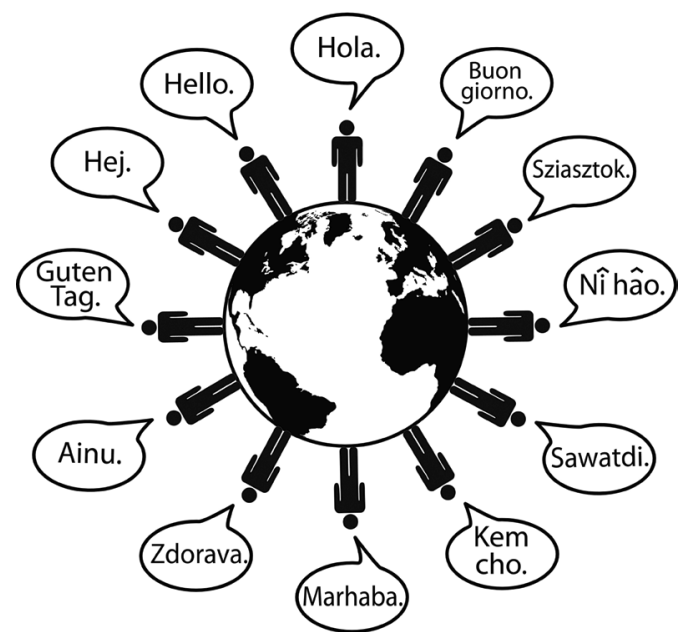

Abb. 1: Sprachen der Welt

Doch gute Rechercheergebnisse sind noch nicht alles: Immer stärker kommt es heute auch darauf an, Suchergebnisse sinnvoll zu bewerten, zu exportieren und effizient zu verwalten, Literatur zu beschaffen, für die eigene Arbeit zu nutzen und natürlich richtig zu zitieren. Mit diesen Themen beschäftigt sich der dritte Teil des Buches (Informationen weiterverarbeiten).

Das Ressourcenverzeichnis am Ende des Bandes weist alle besprochenen Informationsressourcen nach und bietet die Zugangsdaten zu den elektronischen Angeboten. Bei vielen lizenzpflichtigen Datenbanken kann es allerdings nötig sein, einen Rechner Ihrer Universität zu verwenden oder sich mit einem Passwort als Nutzer der Bibliothek zu identifizieren, um Zugriff auf die Inhalte zu bekommen. Den besten Überblick über Ihre Zugangsmöglichkeiten zu Datenbanken gibt Ihnen das Datenbank-Infosystem (DBIS, s. u. S. 31).

Neben den grundlegenden Informationen, die dieses Buch Ihnen geben kann, sollten Sie sich auch mit den spezifischen Angeboten Ihrer lokalen Bibliothek vertraut machen. Nutzen Sie daher die örtlichen Schulungsveranstaltungen und das Informationsmaterial, das auf die lokalen Angebote hinweist.

Vielen Freunden und Bekannten - Studierenden und Dozenten linguistischer Studiengänge ebenso wie Kolleginnen und Kollegen aus dem Bibliothekswesen - bin ich für wichtige Hinweise, Ratschläge und vielfältige Hilfe zu Dank verpflichtet. Namentlich erwähnen möchte ich Prof. Dr. Arnulf Deppermann, Kerstin Gotthardt, Dorothee Graf, Dr. Jochen Haug, Helene Hoffmann, Stephan Hoffmann, Dr. Ulrike Hollender, Ursula Jäcker, Margrit Lauber-Reymann, Prof. Dr. Christer Lindquvist, Doina Oehlmann, Mareike Philipp, Dr. Naoka Werr und Heike Renner-Westermann. Wie immer gebührt der größte Dank meiner Frau Cordula, die das Entstehen dieses Buches in allen Phasen begleitet, gefördert und damit ermöglicht hat.

Widmen möchte ich das vorliegende Buch meiner jüngsten Tochter Magdalena, die während der Arbeit an diesem Band zur Welt kam.

Klaus Gantert, München, Januar 2013 